\title{
POTENCIAL DE EMERGÊNCIA DAS ESPÉCIES DE CLOTALÁRIA JUNCEA E CROTALARIA CHROLEUCA EM FUNÇÃO DA PROFUNDIDADE DE SEMEADURA
}

\author{
GALDINO, Antonia Gorete ${ }^{1}$ \\ CAMARA, Felipe Thomaz ${ }^{1}$ \\ SANTANA, Laudeline Dantas ${ }^{1}$ \\ PINTO, Antonio Alves ${ }^{1}$ \\ SILVA, Josefa Maria ${ }^{2}$
}

\begin{abstract}
RESUMO: O objetivo do presente trabalho foi avaliar a emergência das espécies de Crotalaria juncea e Crotalaria ochroleuca, em condição de campo, em função da profundidade de 1 a $4 \mathrm{~cm}$ semeadura. $\mathrm{O}$ experimento foi conduzido na área experimental do Centro de Ciências Agrárias e da Biodiversidade (CCAB) da Universidade Federal do Cariri (UFCA), Crato-CE. O delineamento inteiramente casualizado, em esquema fatorial $2 \times 4$, com quatro repetições, totalizando 48 observações. O primeiro fator foram as duas espécies de crotalária $(C$. juncea e $C$. ochroleuca) e o segundo foi a profundidade de semeadura $(1 ; 2 ; 3 \mathrm{e} 4 \mathrm{~cm})$. A irrigação utilizada foi por meio de rega (com regadores). Os fatores avaliados foram a porcentagem de emergência aos 7 dias após a semeadura (DAS), o número de dias necessários para estabilizar a emergência (NDE), o índice de velocidade de emergência (IVE) e a altura das plantas aos 7 DAS. É interessante que seja feita a escolha correta da profundidade utilizada, não sendo recomendado o plantio em $1 \mathrm{~cm}$, embora essa profundidade facilite o índice de velocidade de emergência, no entanto, as sementes ficam mais expostas a maiores temperaturas e pode ocorrer rápida falta de água, comprometendo o crescimento e desenvolvimento ideal da planta. A pesquisa mostrou que, para obter a melhor emergência das espécies (C. juncea e $C$. ochroleuca) recomenda-se a profundidade de $4 \mathrm{~cm}$.
\end{abstract}

Palavras-chave: Irrigação. Profundidade. Semeadura. Sementes.

SUMMARY: The objective of the present work was to evaluate the emergence of Crotalaria juncea and Crotalaria ochroleuca species, in field condition, as a function of depth of 1 to $4 \mathrm{~cm}$ sowing. The experiment was conducted in the experimental area of the Center of Agricultural Sciences and Biodiversity (CCAB) of the Federal University of Cariri (UFCA), Crato-CE. The completely randomized design, in a $2 \times 4$ factorial scheme, with four replications, totaling 48 observations. The first factor was the two species of crotalaria (C. juncea and C. ochroleuca) and the second was the seeding depth $(1,2,3$ and $4 \mathrm{~cm})$. The irrigation used was irrigated (with irrigators). The factors evaluated were the emergence percentage at 7 days after sowing (DAS), the number of days needed to stabilize the emergency (NDE), the rate of emergence (IVE) and plant height at 7 DAS. It is interesting to make the correct choice of the depth used, and the planting is not recommended in $1 \mathrm{~cm}$, although this depth facilitates the rate of emergency speed, however, the seeds are more exposed to higher temperatures and can occur rapid water shortage , compromising the ideal growth and development of the plant. The research showed that, to obtain the best emergence of the species (C. juncea and C. ochroleuca) the depth of $4 \mathrm{~cm}$ is recommended.

Keywords: Irrigation. Depth. Sowing. Seeds.

\section{INTRODUÇÃO}

A crotálaria juncea $L$ e $C$. ochroleuca são plantas de crescimento rápido, favorecendo melhor proteção ao solo, chegando a atingir até $3,5 \mathrm{~m}$ de altura. Auxiliam nana fixação de nitrogênio, tem alto potencial de acúmulo de massa seca, favorecendo o fornecimento de matéria orgânica ao solo, podem ser utilizadas como cobertura morta em cultivos consorciados e ainda possuem efeito alelopático, ou seja,

\footnotetext{
${ }^{1}$ Universidade Federal do Cariri - Ciências Agrárias Agronomia

${ }^{2}$ UFC - FITOTECNIA
} 
impede o reduzindo o desenvolvimento de plantas daninhas (MORAES et al., 2006).

A crotálaria juncea $L$ e $C$. ochroleuca pertencem à família das leguminosas, composta por numerosas espécies que apresentam características diversas quanto ao ciclo vegetativo, produção de fitomassa, porte e ainda uma ampla diversidade de exigências em relação a clima e solo. Entendendo as diferenças, mesmo dentro da mesma espécie, são recomendadas para regiões especificas que se adéquam às características da espécie. Por essas razões devem-se procurar combinações desses fatores que atendam às exigências locais, dando-se prioridade às que produzam maior volume de matéria seca, às menos sujeitas a pragas e doenças e às que possuam sementes relativamente uniformes e fáceis de semear, tanto manualmente como por meio de máquinas (CARLOS; FERREIRA, 2001).

Originadas em regiões de clima tropical e subtropical, não suportando temperaturas baixas, são bem resistente à seca, e não tolera encharcamento. Para que a planta se desenvolva com eficiência, é importante que o solo escolhido para a realização do plantio não apresente compactação, já que o sistema radicular das crotalárias não é eficiente na descompactação das camadas do solo (BURLE et al., 2006).

Outras finalidades das culturas é a utilização em áreas infestadas com nematoides, que tem sido um grande problema em áreas de cultivo de hortaliças, principalmente em sistemas de cultivo orgânico por não poder usar químicos, mas, plantas usadas como adubação verde, como a crotalária, tem efeito na redução dos nematóides parasitas de plantas, diminuindo perdas (BRINGEL; SILVA, 2000).

Desta forma, é de suma importância o conhecimento das espécies crotálaria juncea $L$ e $C$. ochroleuca, por causa dos inúmeros benefícios que traz ao solo, tanto física como quimicamente, além de substâncias que são liberadas pela planta e sua ação, tanto em microrganismos como nas invasoras, como por exemplo; o efeito alelopático, que inibe o desenvolvimento das plantas daninhas, e também pela grande capacidade de fixação de nitrogênio (BURLE et al., 2006).

Com relação à semeadura das crotálaria juncea $L$ e $C$. ochroleuca, as empresas que comercializam sementes indicam a profundidade de 2 a $3 \mathrm{~cm}$ como ideais (PIRAI SEMENTES, 2016; SEMEATA, 2016 e BRSEEDS, 2016). Porém, não há dados científicos que evidenciam estas profundidades como sendo as que propiciam melhor emergência das plantas.

Neste contexto, a presente pesquisa teve como objetivo avaliar a emergência das espécies de Crotalária juncea e Crotalária ochroleuca, em condição de campo, em função da profundidade de 1 a 4 cm semeadura.

\section{MATERIAL E MÉTODO}

O estudo foi conduzido no Centro de Ciências Agrárias e da Biodiversidade, na Universidade Federal do Cariri, Crato-CE, localizada na região do Cariri Cearense, situando-se a $442 \mathrm{~m}$ de altitude, com latitude sul de $7^{\circ} 14^{\prime} 3,4^{\prime \prime}$ e longitude oeste de $39^{\circ} 22^{\prime}$ '7,6", em um solo classificado como Argissolo Vermelho Amarelo, conforme classificação do mapa de solos da Funceme (2012), de relevo suave ondulado e textura da camada superficial do solo classificada como franco-arenosa, coberto por espécies espontâneas de pequeno porte.

Foi utilizado o delineamento inteiramente casualizado, em esquema fatorial $2 \times 4$, com quatro repetições, totalizando 48 observações. O primeiro fator foram as duas espécies de crotalária (C. juncea e C. ochroleuca) e o segundo foi a profundidade de semeadura (1;2; 3 e $4 \mathrm{~cm})$.

As sementes utilizadas comerciais vencidas, com validade para o ano de 2013 (Vencida há 3 anos) e sendo comercializadas pela empresa BRSEEDS.

A semeadura foi realizada no campo, sendo semeadas 30 sementes por parcela experimental nas profundidades de $1 \mathrm{a} 4 \mathrm{~cm}$, conforme o tratamento avaliado, e foram avaliados a porcentagem de 
emergência aos 7 dias após a semeadura (DAS), o número de dias necessários para estabilizar a emergência (NDE), o índice de velocidade de emergência (IVE) e a altura das plantas aos 7 DAS.

A porcentagem de emergência aos 7 DAS foi calculada pela relação entre o número de plantas emergidas sete dias após a semeadura e o número de sementes semeadas (30).

O NDE foi obtido por meio da verificação do dia a partir do qual não ocorreram mais emergências, sendo definido desta forma o número de dias necessários para finalizar a emergência das plantas.

O Índice de Velocidade de Emergência (IVE), foi calculado por meio do registro diário do número de plântulas emergidas, com parte aérea formada, até o sétimo dia, quando houve estabilização da emergência para todos tratamentos, sendo calculado pela fórmula proposta por MAGUIRE (1962), em que $\mathrm{IVE}=\mathrm{E} 1 / \mathrm{N} 1+\mathrm{E} 2 / \mathrm{N} 2+\ldots \mathrm{En} / \mathrm{Nn}$, onde $\mathrm{E} 1, \mathrm{E} 2, \ldots \mathrm{En}=$ número de plântulas normais computadas na primeira contagem, na segunda contagem e na última contagem. $\mathrm{N} 1, \mathrm{~N} 2, \ldots \mathrm{Nn}=$ número de dias da semeadura à primeira, segunda e última contagem.

Todos os dados foram submetidos à análise de variância e ao teste de comparação de médias de Tukey a 5\% de probabilidade, sendo realizada análise de regressão para determinar qual foi o menor expoente significativo para o fator profundidade, por ser uma medida quantitativa.

\section{RESULTADO E DISCUSSÃO}

O coeficiente de variação indica a variação dos dados obtidos em relação à média, portanto, de acordo com os dados obtidos na tabela 1, e a classificação proposta por Gomes (1985), o CV da porcentagem de emergência e do IVE, foram médios, ou seja, a dispersão dos dados é média, para o NDE, o CV foi muito alto, acima de $30 \%$, ocorrendo assim uma dispersão muito alta dos dados em relação à media, e para a altura, maior que $20 \%$, teve alta dispersão dos dados em relação à média.

Nas profundidades observou-se uma diferença significativa a 5\% de probabilidade (Tabela 1).

Tabela 1- Síntese da análise de variância para a porcentagem de emergência inicial (\%Ei), a porcentagem de emergência final (\%Ef) número de dias para emergência (NDE) e índice de velocidade de emergência (IVE).

\begin{tabular}{|c|c|c|c|c|}
\hline \multirow{2}{*}{ Fatores } & \multicolumn{4}{|c|}{ Valores de F } \\
\hline & $\% \mathrm{Ei}$ & $\% \mathbf{E f}$ & NDE & IVE \\
\hline Crotalária (C) & $447,2 * *$ & $235,1 * *$ & $126,8 * *$ & $497,2 * *$ \\
\hline Profundidade (P) & $0,89^{\mathrm{NS}}$ & $1,03^{\mathrm{NS}}$ & $3,26 *$ & $7,69 * *$ \\
\hline $\mathrm{CxP}$ & $0,07^{\mathrm{NS}}$ & $0,20^{\mathrm{NS}}$ & $2,94^{\mathrm{NS}}$ & $11,99 * *$ \\
\hline $\mathrm{CV} \%$ & 17,69 & 12,94 & & \\
\hline \multirow{2}{*}{ Crotalária } & \multicolumn{4}{|c|}{ Teste de Médias } \\
\hline & $\% \mathrm{Ei}$ & $\% \mathrm{Ef}$ & $\mathrm{NDE}$ & IVE \\
\hline Juncea & $68,5 \mathrm{a}$ & $69,8 \mathrm{a}$ & $4,62 \mathrm{a}$ & $5,75 \mathrm{a}$ \\
\hline Ochroleuca & $13,9 \mathrm{~b}$ & $33,5 \mathrm{~b}$ & $8,19 \mathrm{~b}$ & $1,68 \mathrm{~b}$ \\
\hline
\end{tabular}

**: significativo $(\mathrm{P}<0,01)$; *: significativo $(\mathrm{P}<0,05)$; NS: não significativo; $\mathrm{CV \%}$ : coeficiente de variação.

Médias seguidas pela mesma letra minúscula na coluna não diferem entre si pelo teste de Tukey a 5\% de probabilidade. 
De acordo com a tabela 1 , notou-se que a porcentagem de emergência final não obteve resposta significativa, com estabilidade nos valores à medida em que se aumentou a profundidade de semeadura, resultados estes que contradizem as indicações atuais para a semeadura em torno de 2 a $3 \mathrm{~cm}$ (PIRAI SEMENTES, 2016; SEMEATA, 2016 e BRSEEDS, 2016). O NDE obteve reposta significativa a $(\mathrm{P}<0,05)$, porem nota-se que no 7 dia de germinação houve uma estabilização na emergência.

O índice de velocidade de emergência (IVE) para Crotalária juncea e C. ochroleuca obtiveram resultados diferentes, pois na $C$. juncea, observou um aumento no índice de velocidade, sendo que o maior IVE registrado foi na profundidade de $1 \mathrm{~cm}$, diferenciado da espécie $C$. ochroleuca, que em todas as profundidades o IVE se manteve constante.

A profundidade de deposição da semente pode afetar a germinação, sendo condicionada pela temperatura, teor de água e tipo de solo, dentre outros fatores. A semente deve ser depositada a uma profundidade que permita um adequado contato com o solo úmido, resultando em elevado percentual de emergência (SILVA et al. 2008).

Figura 2 Índice de velocidade de emergência em função da profundidade de semeadura para as espécies (C. juncea e C. ochroleuca).

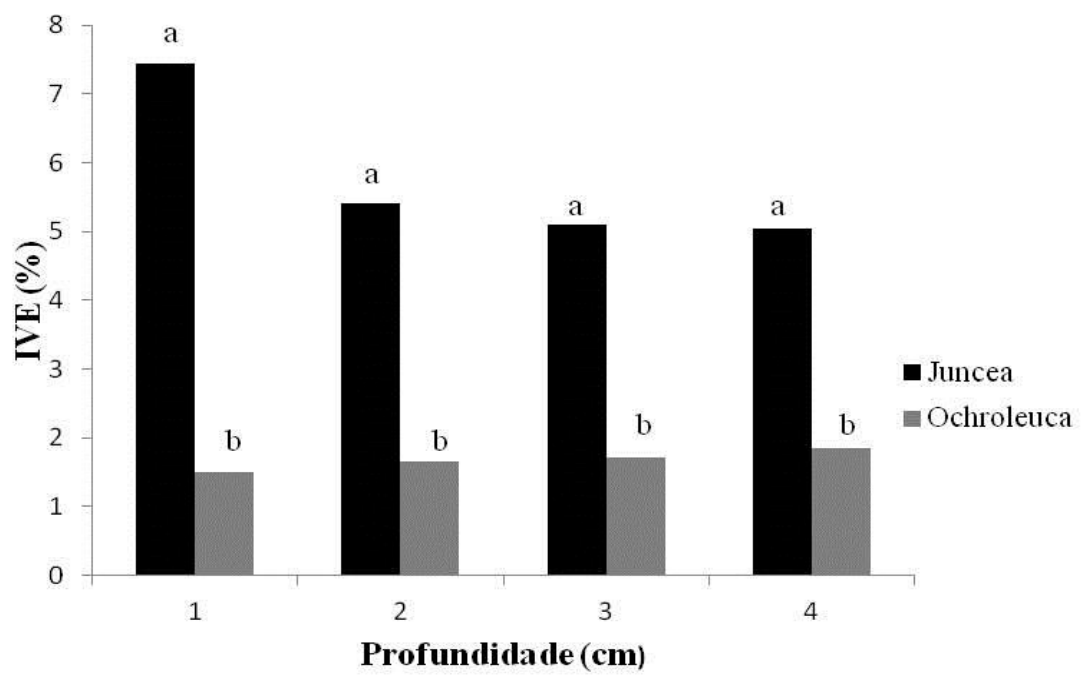

Em comparação com outras leguminosas observou-se que essas duas espécies possui um nível bom de emergência em menores profundidades, por exemplo, segundo a Embrapa (2010) a espécie Ervilha-Forrageira (Pisum sativum L. subespécie arvense), apresenta uma boa emergência nos $20 \mathrm{~cm}$ de profundidades, isso mostra que mesmo sendo cada espécie possui suas características próprias, as diferenças nas profundidades de semeadura estar relacionada também com o tamanho das sementes.

Matos et al (2013) estudando a influência da profundidade de semeadura na germinação do feijão caupi (Vigna unguiculata) nas profundidades de 2,4 e $6 \mathrm{~cm}$, a profundidade de $2 \mathrm{~cm}$ se destacou, apresentando as melhores porcentagens de germinação, sendo que o trabalho objetivou analisar a influência da profundidade de semeadura na germinação de sementes de feijão caupi em três diferentes profundidades. a profundidade de semeadura de $2 \mathrm{~cm}$ inicialmente se destaca, apresentando as melhores porcentagens de germinação, mas que essas porcentagens são atingidas pelos demais tratamentos ao decorrer dos dias de germinação.

Matos et.al (2013) comprovaram o que foi estudado com as espécies de crotalárias, pois em profundidades menores as sementes não têm dificuldades para germinar, mostrando assim que é um 
parâmetro observado em algumas espécies da família leguminosa.

Para a espécie $C$. juncea, o índice de velocidade de emergência IVE, diferenciou estatisticamente com correlação razoável e significativa $(\mathrm{P}<0,01)$ ), porem foi maior em $1 \mathrm{~cm}$, diminuindo esse índice nas outras profundidades.

Desta forma, observou-se que apesar de um pequeno atraso na emergência as maiores profundidades não impedem que as sementes germinem, sendo que as plantas terão uma maior resistência a possíveis veranicos, pois apresentaram um sistema radicular mais profundo na fase inicial.

Figura 3 - Análise de regressão para o índice de velocidade de emergência em função da profundidade de semeadura para as espécies (C. juncea e C. ochroleuca).

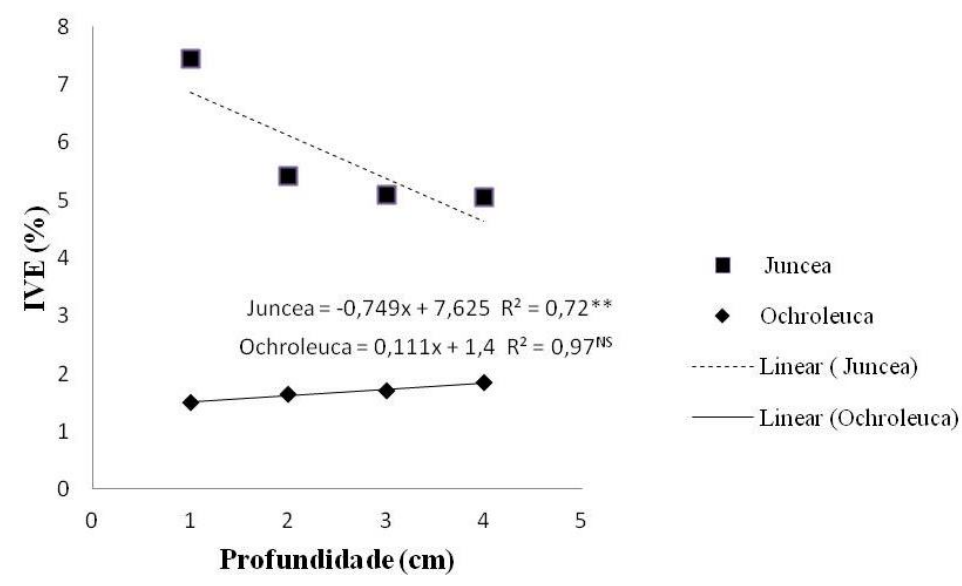

Para a espécie $C$. ochroleuca, observou-se que não houve diferenças estatísticas, mostrando assim, que em qualquer uma das profundidades estudadas o IVE vai se manter o mesmo.

\section{CONCLUSÃO}

Para obter a melhor emergência das espécies (C. juncea e C. ochroleuca) recomenda-se a profundidade de $4 \mathrm{~cm}$.

\section{AGRADECIMENTOS}

Agradecemos ao Programa de Educação Tutorial (PET) pela bolsa de pesquisa, ensino, cultura e extensão, a qual vem nos proporcionando maior conhecimento prático com a realização de pesquisas.

\section{REFERÊNCIAS}

BURLE, M. L. et al. Caracterização das espécies de adubo verde. In: CARVALHO, A. M.; AMABILE, R. F. (Ed.). Cerrado: adubação verde. Planaltina-DF: Embrapa Cerrados, p.71-142, 2006.

CARLOS, B. A.; FERREIRA, M. F. Recomendações técnicas para o uso da adubação verde em solos de tabuleiros costeiros Aracaju: 19 ISSN 1678-1945, Dezembro, 2001. 
EMBRAPA- Empresa Brasileira de Pesquisa Agropecuária. Leguminosas Forrageiras Anuais de

Inverno. Disponível em: http://www.cnpt.embrapa.br/biblio/li/li01-forrageiras/cap10.pdf. Acesso em: 12 Dez.2016.

FUNCEME - Fundação Cearense De Meteorologia E Recursos Hídricos. Levantamento de reconhecimento de média intensidade dos solos da Mesorregião do Sul Cearense. Fortaleza: FUNCEME, 2012.

MORAIS JUNIOR, O.P. de et al. Longevidade de sementes de Crotalária juncea L e Crotalária spectabilis Roth em condições naturais de armazenamento. 2010. Disponível em: http://www.prp2.ueg.br/sic2010/fronteira/arquivos/trabalhos_2009/ciencias_agrarias/sic/longevidade_de_s ementes.pdf. Acesso em: 14 jul. 2016.

MATOS. R. F. et al. Influência Da Profundidade De Semeadura Na Germinação Do Feijão Caupi (Vigna unguiculata). In: CONGRESSO NACIONAL DE FEIJÃO CAUPI, Anais... Recife, 2013.

PIRAÍ Sementes. Crotalária juncea. 2016. Disponível em:

http://www.pirai.com.br/texto-b27-crotalaria_juncea.html>. Acesso em: 15 de jul. 2016.

SEMEATA. Crotalária juncea. Disponível em: <

http://www.semeata.com.br/?sessao=produto\&ver\&id=29>. Acesso em: 15 de jul. 2016.

SILVA, A. A.; CARMELLO, S. M.; NAKAGAWA, J. Germinação e vigor de sementes de Crotalária lanceolata E. Mey. 1. Influência da cor do tegumento e da posição dos frutos na infrutescência. Revista Brasileira de Sementes, Londrina, v. 10, n. 2, p. 67-73, 1988.

SILVA, R. P.et al. Efeito da profundidade de semeadura e de rodas compactadoras submetidas a cargas verticais na temperatura e no teor de água do solo durante a germinação de sementes de milho. Ciência e Agrotecnologia, Lavras, v.32, n.3, p.929-937, 2008. 